\title{
Diagnóstico de Bioseguridad en el Sector Sanitario del Departamento de Bolívar, Norte de Colombia
}

\author{
Elías A. Bedoya $a^{(1)^{\star}}$, Darío D. Sierra ${ }^{(2)}$ Carlos A. Severiche ${ }^{(2)}$ y María de J. Meza ${ }^{(2)}$
}

(1) Fundación Universitaria Tecnológico Comfenalco, Coordinador de investigación del Programa de Tecnología en Seguridad e Higiene Ocupacional, Grupo de Investigación CIPTEC, Cartagena de Indias, Bolívar-Colombia (e-mail: ebedoya@tecnologicocomfenalco.edu.co)

(2) Fundación Universitaria Tecnológico Comfenalco, Facultad de Ingeniería, Programa de Tecnología en Seguridad e Higiene Ocupacional, Cartagena de Indias, Bolívar-Colombia

${ }^{*}$ Autor a quien debe ser dirigida la correspondencia

Recibido Mar. 23, 2017; Aceptado May. 24, 2017; Versión final Jun. 20, 2017, Publicado Oct. 2017

\section{Resumen}

Se diagnosticó las condiciones de bioseguridad en el sector sanitario del departamento de Bolívar, en el Caribe Colombiano. El estudio fue cualitativo, transversal, realizado en 33 instituciones prestadoras de servicios de salud donde se detallaron las condiciones de bioseguridad mediante el uso de listas de chequeo relacionadas con el riesgo biológico, revisión documental y estadísticas de accidentalidad. Se encontraron en promedio 262856 horas hombre trabajadas, siendo los años 2012 y 2013 los más accidentados con riesgo biológico, al registrar 37 y 30 accidentes respectivamente. En las 33 instituciones evaluadas se encontraron en promedio 262856 horas hombre trabajadas, entre los años 2011 y 2013 se generó un promedio de 7.6 accidentes con riesgo biológico, siendo el año 2013 el más accidentado entre las instituciones evaluadas.

\section{Diagnosis of Biosecurity in the Health Sector of the Department of Bolívar, Northern Colombia}

\begin{abstract}
Biosecurity conditions were diagnosed in the health sector of the department of Bolívar, in the Colombian Caribbean. The study was qualitative, cross - sectional, carried out in 33 institutions providing health services that detailed the biosecurity conditions through the use of checklists related to biological risk, documentary review and accident statistics. An average of 262856 man hours were found, with 2012 and 2013 being the years with more injured cases with biological risk, with 37 and 30 accidents respectively. In the 33 institutions that were evaluated, an average of 262856 man hours were found, between the years 2011 and 2013 an average of 7.6 accidents were generated, being the year 2013 the most critical among the evaluated institutions
\end{abstract}




\section{INTRODUCCIÓN}

La seguridad y salud en el trabajo se ha desarrollado durante las últimas décadas, con un enfoque que enfatiza la prevención de los riesgos laborales (accidentes de trabajo y enfermedades) y en la promoción de la salud en el trabajo (Parra, 2003; Yang et al., 2015). De esta forma se ha dado mayor prioridad a mejorar la calidad de vida del trabajador no solamente dentro del trabajo sino fuera del mismo, de esta forma se ha contribuido al desarrollo de la sociedad y del país. Los servicios de salud en los distintos lugares requieren medidas de prevención frente al riesgo de contaminación con agentes biológicos (Parra, 2003; Trincado et al., 2011), para lo cual se requiere adecuado aseo personal (MMWR, 2001), medidas generales de aseo y control de plagas en los locales de trabajo, disponibilidad de agua potable, duchas y casilleros guardarropas. Es importante la información sobre riesgo en personas expuestas, enfermeras, médicos, personal auxiliar e incluso el administrativo (Rodriguez, 2001; Ardila y Muñoz, 2009).

En el servicio de salud al existir desechos humanos o animales, se debe priorizar en el cuidado, por aparición de nuevas enfermedades de extrema gravedad como el síndrome de inmunodeficiencia humana, la enfermedad de las vacas locas o los distintos serotipos de gripe con situaciones de riesgo nuevas y con consecuencias desconocidas (ISTAS, 2015). Donde además se debe priorizar en el usuario de los servicios de salud, requiriendo este una buena calidad de servicio y pronta atención (Vargas y Giraldo, 2014). Para quienes la primera medida preventiva es la información acerca del riesgo específico, la identificación de animales sospechosos y la manipulación de acuerdo a normas escritas (Induambiente, 1999). Lo anterior sumado a los tiempos de atención, se encuentra como otra de las principales problemáticas de la atención al paciente (Martínez et al., 2015).

En algunos casos, la prevención del contagio por riesgo biológico también implica la vacunación (OMS, 2001). La exposición o accidente biológico se puede definir como cualquier trabajador de la salud incluyendo estudiantes y personal en entrenamiento expuesto a sangre o a cualquier otro fluido de personas bajo su cuidado (Montufar et al., 2015; EPINETAC, 2000). Se consideran potencialmente infecciosos los derivados de sangre o líquidos visiblemente contaminados (HICPAC, 2001). Siendo relevante la adecuada planificación de los centros asistenciales y de urgencias dentro de la red sanitaria que adecue el acceso a equipamientos esenciales (Holguín et al., 2017).

Un tercio de los accidentes laborales notificados que afectan a funcionarios de la salud lo constituyen los accidentes corto-punzantes, con serio riesgo de infección por Virus de inmunodeficiencia humana y otros patógenos (MINSAL, 2000; Abreu et al., 2008). El riesgo de exposición continúa en hospitales donde se realizan el mayor tipo de procedimientos invasivos (MINSAL, 2000; OMS, 2006), con mayor contacto en pacientes, coincidiendo con los datos reportados en la bibliografía mundial (Do et al., 2003), concordando con la actividad que la asistencia realiza en este tipo de áreas (Bell, 1997; Min et al., 2005; Kuhar et al., 2013) donde existe un mayor riesgo que se generen accidentes (Bueno, 2007), procedimientos relacionados con agujas en espacio intravascular y lesiones profundas (Kosgeroglu et al., 2003; Dominguez y Trimiño, 2012).

Algunos agentes biológicos también causan lesiones en la piel, heridas supurativas características en algunas enfermedades transmisibles (Beekman et al., 2001), es posible encontrar en algunas actividades laborales un riesgo combinado de lesión de la piel por agente químico, que luego se complica con una infección (Cardo et al., 1997; Aceto, 2015). Existe evidencia suficiente sobre accidentes con elementos cortopunzantes como agujas hipodérmicas que generan pinchazos y contacto con sangre contaminada, transmitiendo infecciones asociadas a VIH, hepatitis C y B (MMWR, 2001). Estudios demuestran la probabilidad de desarrollar enfermedad hepática crónica luego de un accidente de este tipo (Presidencia de la Republica de Colombia, 2014). Son objetivos del presente estudio la identificación de condiciones de bioseguridad el análisis aspectos como la accidentalidad, la gestión de residuos peligrosos y el cumplimiento de aspectos legales entre instituciones sanitarias en el departamento de bolívar, Colombia.

\section{MATERIALES Y METODOS}

Estudio cualitativo de corte descriptivo transversal, desarrollado durante los periodos comprendidos entre diciembre de 2010 y noviembre de 2013. Consistió en analizar registros de accidentalidad en documentos relacionados con la gestión de residuos hospitalarios y similares junto a técnicas de observación no participativa y registro de información en 33 instituciones distribuidas a lo largo del departamento de Bolívar, iniciando en Cartagena y finalizando en el municipio de Altos del Rosario. Los criterios de inclusión fueron las instituciones prestadoras de salud (IPS) con información actualizada sobre manejo de residuos y accidentalidad general y biológica. Se excluyeron IPS sin información sobre accidentalidad ni datos sobre gestión de residuos hospitalarios.

Se inspeccionaron áreas de trabajo, diligenciaron listas de chequeo sobre condiciones de bioseguridad y manejo de residuos, conforme a las disposiciones del ministerio de salud colombiano, actualizados conforme 
a los estándares de del sistema de garantía de la calidad en salud, útiles y homologados a nivel internacional tomando en cuenta para su elaboración a las directrices del manual para la gestión integral de residuos generados en la atención en salud y otras actividades detectando operaciones en relación al riesgo biológico, volumen de residuos producidos y condiciones de trabajo con sustancias biológicas con potencial para generar accidentes de trabajo y registro de información para análisis de estas. Se revisó posteriormente los documentos para el cumplimiento de las exigencias legales en instituciones de salud en cuanto a la gestión de residuos hospitalarios. Se calcularon datos de accidentalidad organizados y tabulados, se procedió a interpretar la información relacionada con bioseguridad, usando el programa Excel versión 2013 para el manejo de la base de datos y generación de estadísticas básicas (promedios, porcentajes, horas hombre trabajadas e indicies de accidentalidad) en cuadros relacionados, dichos resultados permiten ser utilizados como parámetro comparativo con otras instituciones prestadoras de servicios de salud a nivel departamental, nacional e internacional referente a condiciones biológicas y de accidentalidad. En cuanto a las Consideraciones éticas, este es un estudio de riesgo mínimo como los estipula la Resolución No. 008430 de 1993 del Ministerio de Salud y en la Ley 84 de 1989.

\section{RESULTADOS}

Se visitaron 33 IPS del departamento de Bolívar, las cuales fueron seleccionadas aleatoriamente, de las cuales $78.7 \%$ (26 de 33) son de primer nivel de complejidad 9.09\% (3 de 33) de segundo nivel y 12,2\% (4 de 33) de tercer nivel. Un $12.1 \%$ (4 de 33) se encuentran en el área urbana de Cartagena y $87,8 \%$ (29 de 33) restantes en municipios del departamento de Bolívar. Se evaluaron registros de accidentalidad en 2938 trabajadores distribuidos entre trabajadores asistencias con un $61,6 \%$ (1810 de 2938) por ende más susceptibles al riesgo biológico en las IPS objeto del estudio un $22,2 \%$ (653 de 2938) son operativos y $16,1 \%$ administrativos (475 de 2938).

El promedio de horas hombre trabajadas en estas instituciones asciende a 262856 por año. Durante los años 2010 a 2013 se registraron en promedio 23,5 (Desviación Estándar 15.6) accidentes no biológicos, mientras que por sustancias biológicas contaminadas el número promedio llegó a 20 (Desviación Estándar S 1.9). Por cada 100.000 horas hombre trabajadas en las IPS del departamento de Bolívar se generaron en promedio 8,7 accidentes de origen no biológico entre los años 2010 a 2013 como se muestra en la Tabla 1, mientras en los mismos años se generaron en promedio 7,6 accidentes de origen biológico respectiva-mente.

Tabla 1: Características de las IPS evaluadas

\begin{tabular}{|c|c|c|c|}
\hline \multirow{2}{*}{\multicolumn{2}{|c|}{ Capacidad instalada en camas }} & Cantidad & Promedio por IPS \\
\hline & & 806 & 47,41 \\
\hline \multicolumn{2}{|c|}{ Empleados administrativos } & 475 & 28,79 \\
\hline \multicolumn{2}{|c|}{ Empleados asistenciales } & 1810 & 106,47 \\
\hline \multicolumn{2}{|c|}{ Empleados servicios generales } & 653 & 38,41 \\
\hline \multirow{4}{*}{ Accidentes de origen biológico } & 2010 & 1 & 0,13 \\
\hline & 2011 & 12 & 1,33 \\
\hline & 2012 & 37 & 1,32 \\
\hline & 2013 & 30 & 0,97 \\
\hline Horas hombre trabajadas & $2010-2013$ & \multicolumn{2}{|c|}{$262.856,86$} \\
\hline
\end{tabular}

Por cada 100.000 horas hombre trabajadas se generaron 80 accidentes relacionados con residuo biológico tal como se ilustra en la Tabla 2, donde $93.7 \%$ (75 de 80) fueron con material cortopunzante, sobresaliendo que la principal causa es la mala manipulación de las agujas y reencapsulado de biseles en capuchones de las jeringas, cortes accidentales con bisturí y lancetas; $5 \%$ de los accidentes ocurrió con residuo biosanitarios (4 de 80) principalmente por manejo incorrecto de guantes y fluidos contaminados los cuales generaron salpicaduras junto a contacto accidental por vía oral y mucosas mientras un $1.25 \%$ ( 1 de 80 ) por residuos anatomopatológicos o restos diseccionados de pacientes.

Otro de los hallazgos relevantes de esta revisión se detalla en la Tabla 3, están relacionados con los tiempos de recolección de los residuos donde según los registros existieron meses en los cuales los residuos no eran recolectados incrementando el volumen de estos y serias variaciones en las cantidades analizadas. 
Tabla 2: Producción de residuos Vs accidentes con riesgo Biológico Vs IFAT

\begin{tabular}{|l|c|c|c|c|}
\hline $\begin{array}{l}\text { Kilos totales de } \\
\text { residuos producido }\end{array}$ & $\begin{array}{c}\text { Promedio } \\
\text { kilogramos } \\
\text { generados }\end{array}$ & $\begin{array}{c}\text { Accidentes } \\
\text { generados }\end{array}$ & $\begin{array}{c}\text { Promedio } \\
\text { accidentes por tipo } \\
\text { de residuo }\end{array}$ & $\begin{array}{c}\text { IFAT Riesgo } \\
\text { biológico }\end{array}$ \\
\hline Cortopunzante & 1813,25 & 75 & 4,13 & 28,53 \\
\hline Anatomopatologico & 1670,25 & 1 & 0,05 & 0,38 \\
\hline Químico & 2753,5 & 0 & 0 & 0 \\
\hline Biosanitario & 107822,47 & 4 & 0,003 & 1,52 \\
\hline Biológico & 19 & 0 & 0 & 0 \\
\hline Totales & 114078,47 & 80 & 0,07012717 & 30,43 \\
\hline HHT= & \multicolumn{2}{|c|}{262856,8613} & $\mathrm{k}=100.000$ \\
\hline
\end{tabular}

Tabla 3: Aspectos legales en las IPS relacionados con riesgo Biológico

\begin{tabular}{|l|c|}
\hline Cumplimiento de aspectos legales en las IPS relacionados con riesgo Biológico & Cantidad n (\%) \\
\hline Formulario especial de inscripción de prestador de servicios de salud & $32(96,97)$ \\
\hline Formato de verificación de condiciones (visita de verificación) & $33(96,97)$ \\
\hline GAGAS (grupo administrativo de gestión ambiental y sanitaria) & $24(72,73)$ \\
\hline Contrato con recolector de residuos con licencia ambiental & $29(87,88)$ \\
\hline PGIRHS (Plan de gestión integral de residuos hospitalarios y similares) & $22(66,67)$ \\
\hline Evidencias PIGRHS & $14(42,42)$ \\
\hline Indicadores relacionados con el PIGRHS & $9(27,27)$ \\
\hline
\end{tabular}

Todas las instituciones visitadas (33 de 33) indicaron estar verificadas ante el ente de vigilancia y control, aunque una de estas no contaba con el formulario de inscripción que lo acreditara y sólo $87 \%$ (29 de 33) poseen certificado de verificación, comité de ética y contrato de recolección de residuos. Dentro de las principales carencias documentales se encuentran los indicadores del Plan de gestión integral de residuos hospitalarios y similares PGIRHS, donde sólo 27\%(9 de 33) de las IPS cuentan con estos y las demás evidencias, solo eran soportadas en el 42,42\% (14 de 33 ) de las instituciones.

En relación con las condiciones locativas de los lugares de trabajo se evidenciaron falencias especialmente en los depósitos de recolección de residuos, debido a la inexistencia de las condiciones consagradas en el manual de gestión de residuos hospitalarios o similares. En las instituciones evaluadas el $96,9 \%$ de estas (32 de 33) disponen de un área para el almacenamiento para residuos peligrosos y solo una institución adolece de esta área. Sin embargo las condiciones locativas de estas áreas no cumplen con las condiciones citadas en el manual de gestión de residuos hospitalarios y similares colombiano, debido a que en la mayoría de estas áreas existe , falta la señalización relacionada con el riesgo biológico; además no se encuentra una debida seguridad física que evite el ingreso de personal ajeno a la custodia de los residuos (puertas con candado o cerradura, angeos metálicos en las ventanas que eviten el ingreso de animales y otros vectores).

El 36,36\% de las instituciones evaluadas (12 de 33) no disponen de ruta crítica para la recolección de residuos peligrosos debidamente señalizados, además se evidenciaron rutas incompletas, mal señalizadas e incluso sin sentido (que no conducen a ningún lado) y deben ser intervenidas para una mejora disposición y aprovechamiento de los espacios destinados a la señalización. El 66,66\% de las instituciones visitadas (22 de 33) no disponen de carro rodante para la evacuación de los residuos de las diferentes áreas, lo que indica insuficiencia para evacuar estos residuos de forma segura, por se hace susceptible a derrames y accidentes durante el recorrido hasta el área de almacenamiento temporal. En igual proporción se encontraron Instituciones donde la recolección de residuos es manual, faltando el uso del carro rodante; en $81,81 \%$ de las instituciones no existe un lugar adecuado para el almacenamiento, lavado, limpieza y desinfección de los recipientes, vehículos de recolección y demás implementos utilizados, lo cual predispone al trabajador encargado a salpicaduras, contacto con líquidos contaminados y demás elementos y situaciones donde se pueden generar daños al trabajador con contacto o exposición de elementos biológicos contaminados, encontrándose mala disposición final de los residuos, colocación de estos a la intemperie, sin rotulación ni salvaguarda de los elementos recolectados, exponiendo los residuos a los vectores existentes en el área ya sean animales o elementos de diferente naturaleza. De igual forma los recipientes utilizados en la recolección de los cortopunzantes que no cumplen con las condiciones mínimas del manual de gestión de residuos 
hospitalarios, debido que estos son improvisados (botellas de plástico, gaseosa, galones de cloro y otros) sin las características mínimas de la norma (contenedor de barrera sólida, con tapa, rótulo y aperturas requeridas para el ingreso de los cortopunzantes (agujas, bisturí, lancetas y otros).

Otra de las consecuencias de la mala disposición de los residuos resalta en el 90,90\% (30 de 33) de las instituciones, por la presencia de anatomopatológicos, expuestos, mal conservados y contaminando las áreas de almacenamiento, debido a que estos requieren un dispositivo de congelación (refrigerador de uso exclusivo) que permita solidificar el anatomopatológico haciéndolo compacto y evitando derrames ocasionales que generen contaminación de áreas e infecciones recurrentes en los distintos espacios, como también una entrega segura del residuo al recolector. Además los residuos hospitalarios peligrosos no son colocados en canastillas o recipientes rígidos, en contravención al manual de gestión de residuos hospitalarios, al colocar los residuos en el suelo y se acarrea una mayor probabilidad de accidente entre los operarios de la recolección y traslado de estos al área de almacenamiento de los residuos, además genera mayor desorden y dificultad la tarea del pesaje la cual es una labor más dispendiosa y más aún en aquellas instituciones donde no existe un peso para tal fin, donde se depende del criterio del recolector quien generalmente lo aporta y cuando esto no ocurre se apela a un cálculo arbitrario por parte de este.

Por características del medio (aspectos geográficos y dificultad de acceso) en las organizaciones prestadoras de servicios de salud objeto de estudio el 33,33\% (11 de 33) de estas no existe recolección de los residuos peligrosos en intervalos menores a 7 días, tiempo en el cual estos pueden desencadenar en situaciones de degradación y mayor contaminación en las áreas asistenciales, aludiendo dificultades geográficas y falta de vehículos acondicionados para el traslado de los residuos, 11 instituciones evidenciaron un almacenamiento inadecuado (aglomeración, de bolsas en canecos y en el suelo, derribo de bolsas e imposibilidad para cerrar el depósito por exceso de residuos) lo que va en claro incumplimiento de las disposiciones de seguridad requeridas para el almacenamiento de los residuos hospitalarios. De las Instituciones evaluadas el 60,60\% (20 de 33) de estas se localizan en lugares sin riesgo continuo e inminente de desastres naturales, o con áreas adyacentes con riesgos de salubridad graves e incorregibles que impiden mantener las condiciones internas de la institución y acordes con los manuales de Bioseguridad.

\section{DISCUSIÓN}

Dentro de los hallazgos de la investigación resaltan deficiencias en el orden y manejo de las áreas de trabajo con residuos biológicos lo que es un agravante contradictorio con lo expuesto por el Center for Disease and Prevention en 2001, pues las disposiciones locativas dificultan el propósito primario de bioseguridad (HICPAC, 2001) debido a que en la mayoría de las IPS evaluadas, estas adolecen de medidas generales de aseo y control de plagas en los locales de trabajo, duchas y casilleros guardarropas. Lo que, inicialmente propiciando condiciones adversas para una verdadera gestión de bioseguridad, facilitando condiciones para la aparición de accidentes de tipo biológico y general. En cuanto a las capacitación e información sobre riesgo biológico específico, se encontró que esta medida de prevención es escasa entre las instituciones; omitiéndose las disposiciones legales que rigen sobre este tema en el país y que tiene a los hospitales como principales actores (Induambiente, 1999), Las instituciones no promueven como medida propia la vacunación de sus trabajadores y solo se limitan a exigir que los aspirantes a ingresar a los cargos estén ya inmunizados (OMS, 2001) contrariamente a lo dispuesto a la organización mundial de la salud donde la inmunización es una prioridad que debe ser aplicada con especial dedicación debido a que son estrategias de prevención de la aparición de enfermedades infectocontagiosas especialmente en niños, también riñendo con los que el programa ampliado de inmunización promueve.

Coincidiendo con Abreu et al. (2008) y la organización mundial de la salud (OMS, 2006), al indicar que el tipo de accidente biológico que afecta a los funcionarios de los servicios sanitarios es en su mayoría el constituido por corto-punzantes, con serio riesgo de infección por VHB, VHC, VIH y otros patógenos, debido a que en la evaluación 75 de los 80 accidentes con riesgo biológico correspondieron a cortopunzantes. Confirmando a la actividad de asistencia médica y de enfermería como las más propensas a este tipo de accidentes teniendo en cuenta que estos profesionales los que más desarrollan actividades de aplicación de medicamentos, limpieza y cuidado de pacientes (Do et al., 2003) y concordando con las investigaciones de Bell (1997) y Kuhar et al. 2013, al referir que donde exista contactos con sangre, agujas visiblemente contaminadas existe por ende un mayor riesgo que se generen accidentes, siendo las agujas y los procedimientos relacionados con estas en espacio intravascular y lesiones profundas las más lesivas para los trabajadores del servicio de salud (Bueno, 2007) como también se coincidió con los resultados del estudio adelantado por Cardo et al. (1997) quienes afirman encontrar evidencia sobre los pinchazos con agujas hipodérmicas con sangre contaminada, los cuales pueden transmitir la infección por $\mathrm{VIH}$, hepatitis C y $\mathrm{B}$. Lo anterior en contravención a lo reglamentado en la legislación colombiana tratante sobre gestión integral de residuos hospitalarios y similares (Presidencia de la Republica de Colombia, 2014). 


\section{CONCLUSIONES}

En las 33 IPS evaluadas, se encontraron en promedio 262856 horas hombre trabajadas, entre los años 2011 y 2013 se generó un promedio de 8.7 accidentes no biológicos y 7.6 con riesgo biológico, siendo el año 2013 el más accidentado entre las instituciones evaluadas. Se concluye que las capacitaciones deben ser de tipo práctico para tratar de revaluar algunas prácticas subestandar que enfermeras y médicos tienen de forma errada (especialmente la "costumbre" de reenfundar el capuchón de la aguja), una de las más frecuentes generadoras de accidentes entres los afectados consultados en el estudio, esto con el fin de reducir la tasa de accidentes de origen biológico por horas hombre trabajadas.

\section{REFERENCIAS}

Abreu, O., Rodríguez, O., Pérez, E., González, M. Bioseguridad: su comportamiento. Rev. Arch. Méd. Camagüey, 12(5), 27-31 (2008)

Ardila, A., y Muñoz, A. Bioseguridad con énfasis en contaminantes biológicos en trabajadores de la salud. Ciência \& Saúde Coletiva, 14(6), 2135-2141 (2009)

Beekmann, S., Vaughn, T., McCoy, K., Ferguson K., Torner J., Woolson R. y Doebbeling, B. Hospital bloodborne pathogens programs: Program characteristics and blood and body fluid exposure rates Infection Control \& Hospital Epidemiology, Publicación en línea: 02 enero de 2015, DOI: https://doi.org/10.1086/501867, 22 (2) 73-82 (2001)

Bell, D. Occupational risk of human immunodeficiency virus infection in healthcare workers: An overview. Am J. Med., 102(5B), 9-15 (1997)

Bueno, L., Álvarez, M., Guanche, H., García, E. Prevalencia de lesiones por objetos cortopunzantes en el personal de enfermería de unidades de terapia quirúrgicas. Rev. Cubana Hig. Epidemiol., 45(2), 1561-3003 (2007)

Cardo, D.M., Culver, D.H., Ciesielski, C.A., Srivastava, P.U., Marcus, R. y otros cinco autores. A case-control study of HIV seroconversión in health care workers after percutaneaus exposure, doi: 10.1056/NEJM199711203372101, N. Engl. J. Med. (en línea) 337, 1485-1490 (1997)

Center for Disease and Prevention. Update U.S. Public Health Service. Guidelines for management of occupational exposures to HBV, HCV, and HIV and recommendations for post exposure prophylaxis (2001)

Centers for Disease Control and Prevention. Healthcare Infection Control Practices Advisory Committe (HICPAC). Draft Guideline for Environmental Infection Control in Healthcare Facilities (2001)

Composición de Residuos Sólidos a Nivel Nacional. Porcentajes anuales (datos preliminares) Fuente: Directorio Induambiente 99/2000. Guía de Descontaminación Industrial y Recursos Energéticos. Santiago. 230 (1999)

Do, A., Cielsielski, C., Metler, R., Hammett, T., Li, J. y Fleming, P. Occupationally acquired human immunodeficiency virus (HIV) infection: National case surveillance data during 20 years of the HIV epidemic in the United States. Infect. Control Hosp. Epidemiol., 24(2), 86-96 (2003)

Domínguez, Y., Trimiño, A. Evaluación de la calidad de la bioseguridad en el hospital clinicoquirúrgico "Joaquín Albarrán", La Habana, Revista Cubana de Higiene y Epidemiología, 50(1), 67-75 (2012)

Guidelines for the management of occupational exposures to HBV, HCV and HIV and recommendations for postexposure prophylaxis. MMWR Morb Mortal Wkly Rep, 50 (RR-11) 1-52 (2001)

Helen, Aceto. Chapter 29 - Biosecurity in Hospitals, In Robinson's Current Therapy in Equine Medicine, $7^{\text {th }}$ Ed. Edited by Kim A. Sprayberry and N. Edward Robinson, W.B. Saunders, St. Louis, 125-129 (2015)

Holguín, J.M., Escobar, D.A., y Tamayo, J.A. Emergency Attention versus Transport Infrastructure. An analysis of accessibility focused in social inequalities. Study Case: Manizales, Colombia. Información Tecnológica, 28(2), 125-134, Disponible en: https://goo.gl/acm7g8 (2017) 
ISTAS, Instituto Sindical de Trabajo, Ambiente y Salud. Peligros y riesgos laborales. Riesgo biológico. La prevención del riesgo biológico en el trabajo debe evitar las exposiciones laborales capaces de originar algún tipo de infección, alergia o toxicidad. Disponible en: https://goo.gl/eG8TKW. Acceso: 2 de Septiembre (2015)

Kosgeroglu, N., Ayrance U., Vandareli, E., Dincer S. Occupational exposure to hepatitis infection among Turkish nurses: Frequency of needle exposure, sharps injuries and vaccination. Doi: 10.1017/S0950268803001407, Epidemiol Infect., 132, 27-33 (2003)

Kuhar, D., Henderson, D., Struble, K., Heneine, W., Thomas, V., Cheever, L. Updated US Public Health Service Guidelines for the management of occupational exposures to human immunodeficiency virus and recommendations for postexposure prophylaxis. Infect Control Hosp. Epidemiol., 34(9), 875-892 (2013)

Lianping, Y., Chaojie, L., Adamm, J., y Zhang, X. Organizational barriers associated with the implementation of national essential medicines policy: A cross-sectional study of township hospitals in China, Social Science \& Medicine, 145, 201-208 (2015)

Martínez, P., Martínez, J., Nuño, P., y Cavazos, J. Improvement of Patient Care Time in an Emergency Department through the Application of Lean Manufacturing. Information Tecnológica, 26(6), 187-198 (2015)

Min, Y.S., Lim, Hs., Lee, K., Jung, C., Cheong, H.K. A study on the epidemiologic characteristics of scrub typhus in Gyeongsangbuk-do, 1999-2001. Korean J. Epidemiol., 27, 70-79 (2005)

Montufar, F., Villa, J., Madrid, C., Díaz, L., Vega, J., Vélez, J., Zuleta, J. y Grupo de Investigación GIERI. Infección por VIH posterior a exposición ocupacional de riesgo biológico en trabajadores de la salud. DOI: 10.1016/j.infect.2014.04.003, Infectio (en línea), Disponible en: https://goo.gl/XC9fSK, 19(1), 31-34 (2015)

Norma $N^{\circ} 48$ de manejo post-exposición laboral a sangre en el contexto de la prevención de la infección por VIH. MINSAL Octubre (2000)

Parra, M. Conceptos básicos en salud laboral, Santiago, Oficina Internacional del Trabajo (2003)

Presidencia de la República de Colombia. Decreto 351, 19 de febrero de 2014, Por el cual se reglamenta la gestión integral de los residuos generados en la atención en salud y otras actividades. Santa Fe de Bogotá: Diario Oficial 49069,1-2 (2015)

Rodríguez, J., Temas de bioseguridad. La Habana: Editorial «Félix Varela»; p. 338 (2001)

Sociedad Española de Medicina Preventiva, Salud Pública e Higiene. Proyecto EPINETAC (1998-2000)

Trincado, M., Ramos, I., Vázquez, Y. y Guillén, M., Evaluación de las normas de bioseguridad en el servicio de hemodiálisis del Instituto de Nefrología "Dr. Abelardo Buch López", 2009. Revista Cubana de Higiene y Epidemiología, 49(3), 356-372 (2011)

Vargas, J.M. y Giraldo, J.A. Prediction Model of Costs in Health Services Supported in Discrete Simulation. Información Tecnológica, 25(4), 175-184 (2014)

World Health Organization. Review of Health Impacts from Microbiological Hazards in Health-Care Wastes (2001)

World Health Organization. The World Health Report. Working together for health. Geneva: World Health Organization (2006) 
